\title{
A METHOD TO CALCULATE PRESSURE PEAKS BASED ON FREE SURFACE SHAPE: PRELIMINARY RESULTS
}

\author{
Cyril Mokrani ${ }^{1}$, Stéphane Abadie ${ }^{1}$
}

\begin{abstract}
Impulsive loads are difficult to predict due to the extremely non linear response to incident wave conditions. This behavior is related to air dynamics and local wave shape. The present study focuses on the latter.We propose a new method to predict pressure peaks generated during breaking wave impacts. In this method, the plunging jet is assimilated to two equivalent triangular jets (wedge impact) with variable inclination in time. The basic case of wedge impact is first studied in the paper. Semi empirical laws relating pressure peak and incident angle are derived based on numerical results obtained with a Navier-Stokes model . The more general case of a breaking wave is then investigated. By making an analogy with the wedge impact case and inverting the relation obtained before, we computed the location where the equivalent angles have to be taken on the free surface. We show that these points correspond to the minimum curvature section on the free surface. In another simulation of a breaking wave, we finally show how the relation can be applied to give a first approximation of the pressure peak only based on the free surface local shape.
\end{abstract}

Keywords: vertical break-water, pressure peak, breaking wave, wedge, impact

\section{INTRODUCTION}

Impulsive loads generated by breaking waves are known to greatly affect structure stability. They are characterized by high pressure peaks occurring during relatively short times. Experimental observations report a wide scatter in pressure peak measurement. Consequently, impulsive loads are usually predicted by empirical formulae (Nagai 1973) or integrated like in pressure impulse model (Cooker and Peregrine 1995).

Two main processes are involved in that phenomenon. Air compressibility greatly affects impact dynamics. Several mechanisms have been experimentally observed (e.g., air pocket oscillation, air leakage, expulsion, cushioning effects, etc.) The free surface local shape at impact also has a great influence on pressure peak (Whillock 1987). This has been observed in lots of studies devoted to sloshing or hull slamming (Greco 2001). Hull and Muller (2002) observed a great variability in wave impact measurement (even with weak air entrapment). More recently, Lugni et al. (2006) showed that two millimeters high instabilities on interface profiles could produce a $30 \mathrm{kPa}$ variation in pressure peaks. Finally, plunging jet shape determines air pocket dimensions and consequently, all subsequent entrapment effects.

In our study, we use numerical simulations to provide a way to approximate the instantaneous pressure peak only base on the free surface shape. The method is described hereafter.

\section{TRIANGLE WEDGE IMPACT}

Our aim is somehow to predict the impact of a plunging jet given the geometrical properties of the interface. For that purpose, we propose to use the particular case of a triangular wedge impacting a wall (cf. Figure 1a). This is the most basic impact flow as in this case, the geometrical properties of the free surface are reduced to one single data (i.e., the angle $\alpha$ ). During the impact, the free surface moves up the wall. The highest position of the free surface on the wall is denoted $y_{2}$ in the following. The pressure peak is located at position $y_{0}$, which also moves up the wall. Greco (2001) shows that corresponding solutions are self-similar due to the absence of a characteristic length scale.
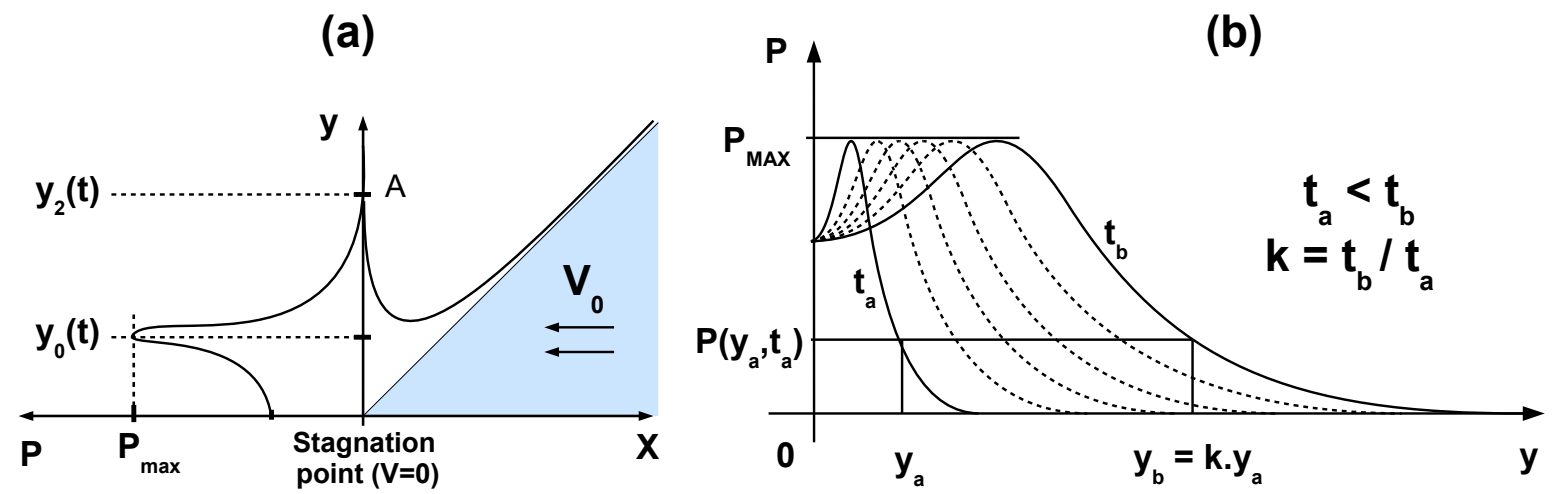

Figure 1: (a) Symmetrical triangular wedge impact - (b) : Geometrical stretching property of solution

\footnotetext{
${ }^{1}$ S.I.A.M.E. Laboratory, Allée du Parc Montaury, 64600 Anglet, France
} 
Therefore, solutions can be expressed with stretched coordinates, and pressure profile on the wall follows an homothetic transformation (i.e., dilatation) as presented on figure 1b. In particular, the maximum pressure keeps constant in time and both the peak position and the point A move with constant velocity. Thus, a specific impact dynamics which does not vary with time is associated to a particular value of $\alpha$.

(a)

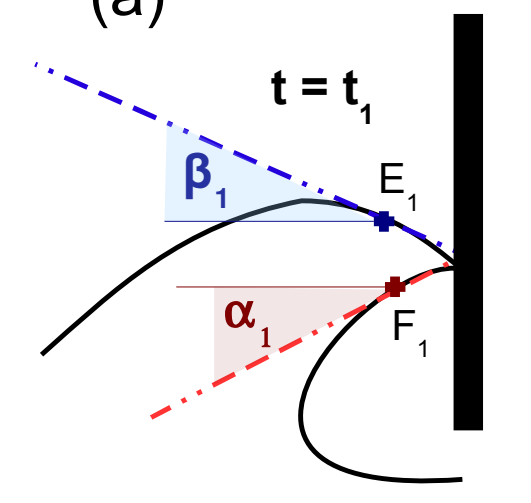

(b)

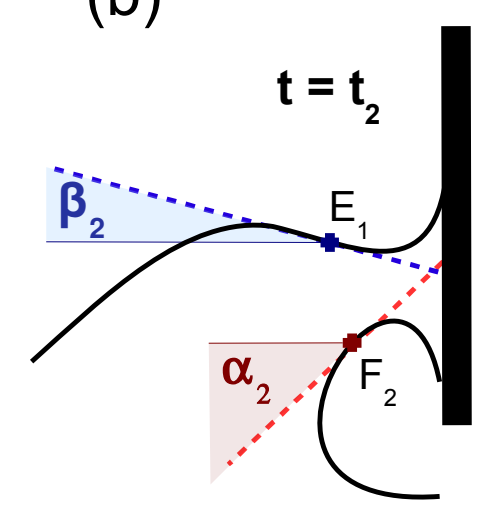

Figure 2: Approach proposed to investigate the dynamical process

Given that, the main idea of our work is to use this wedge flow in order to interpret the more general breaking wave jet impact. More specifically, we propose to interpret the breaking wave jet impact as a succession of time evolving triangular jet impacts. Figure 2 illustrates this idea. The plunging wave jet is assimilated to two triangular jets parametrized by angles $\alpha$ and $\beta$. The value of $\alpha$ and $\beta$ varies in time. But we think that for a given value of $\alpha$ and $\beta$, the breaking wave impact should not be to far away from the one of the corresponding wedge impact. The locations where the angles are taken on the jet are so far arbitrary (cf. Figure 2, points $E_{1}, E_{2}, F_{1}, F_{2}$ ). However, figure 1 a shows that in the wedge case, the angle $\alpha$ is a measure of the free surface slope right upstream the free surface through. For the wave case (figure 2b), it seems logical to place the equivalent angles in the most linear section of the free surface right upstream the trough. In section 3 we will propose an inverse approach to determine the optimum position of the angles.

In the following, we focus on the evolution of three particular parameters : the peak magnitude, the peak position, and the time position of the point A (cf. figure 1a). The objective is to obtain a set of equations linking these parameters.

The wedge impact can be described by the 2D incompressible Navier-Stokes equations (NSE), in which gravity and viscous effects are neglected. Mokrani (2012) shows that this equation can be written in the self-similar domain in the following form :

$$
G^{\prime}(\eta)=F^{\prime}(\eta)(\eta-F(\eta))
$$

with $\eta=\frac{y}{s}, P(y, t)=P_{0} \cdot G(\eta), V(y, t)=V_{0} \cdot F(\eta), s=V_{0} t$ and $P_{0}=\rho V_{0}^{2}$. The peak position and peak magnitude are denoted $\eta_{0}$ and $G\left(\eta_{0}\right)$ respectively in the self-similar plan. To calculate the pressure peak magnitude, it is necessary to integrate equation (1) according to the vertical strectched coordinate $\eta$. However, the pressure peak magnitude is obtained according to the vertical velocity $F(\eta)$ and the latter remains unknown. Consequently, more assumption on $F$ is needed. Several studies in the literature propose to neglect rotational effects in order to consider the potential function (Cumberbatch (1960), Dobrovol'skaya (1969)). The problem is complex to solve analytically. Instead, in the present study, semi-empirical results are developed from numerical simulations performed with the code THETIS. This model solves the incompressible Navier-Stokes equations in 1-fluid formulation with a VOF interface tracking (Yougs et al. 1982). In our study, water an air are simulated but compressibility effects are not included as we focus more on the interface shape problem than the air effect. An improved smoothing algorithm (Pianet et al. 2010) is used in order to control the interface thickness. This allows to deal with interface fragmentation generated in violent impacts. THETIS has been extensively validated in wave related studies among (Abadie et al. 1998, Lubin et al. 2006). In the following, it is tested on the symmetrical wedge impact.

The first step of the approach is the validation of the numerical model in the wedge impact problem 


\section{Validation of the numerical model}

As previously mentioned, self-similar properties ensures that the peak magnitude keeps stable in time in this problem. Likewise, both peak position and point $A$ move with a constant velocity. This will be the first thing to verify in the numerical results. The wedge impact has also been studied by Wu (2007) using analytical and BEM methods. Wu (2007) gives pressure profiles on the wall and interface deformations in the self-similar plan for three particular values of $\alpha$. These results are also used as reference in the present study.

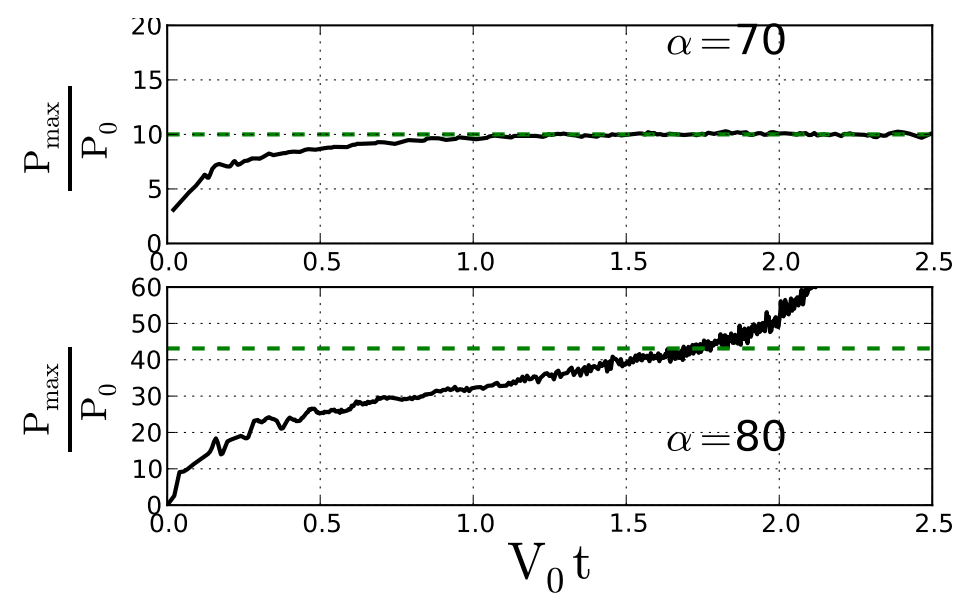

Figure 3: Evolution of the peak magnitude for $\alpha=70^{\circ}$ and $80^{\circ}$ - Comparison with Wu (2007)'s results (- -)

Numerical simulations of wedge impacts have been carried out for $\alpha$ ranging from $10^{\circ}$ to $80^{\circ}$ with step $\Delta \alpha=10^{\circ}$. Results for the case $\alpha=70^{\circ}$ is presented on figure 3. The peak magnitude converges toward the expected value. An accuracy of $1 \%$ is reached after $V_{0} t=2$. Obviously, there is a transitory stage in the simulated flow, which in theory should not exist. This transitory period is shown to increase logically with $\alpha$, as the flow is more and more violent and therefore more difficult to solve. For $10^{\circ}<\alpha<70^{\circ}$, pressure peaks are also stable in time and their values in good agreement with results from Wu (2007). On the contrary, for the highest angle (i.e., $80 \alpha$ ), the pressure peak keeps increasing and does not stabilize at any time in contradiction with the self similar theory. The result can be slightly improved by using finer mesh grids and smaller time steps but so far, a constant pressure peak is still not reached. So, for the highest inclination tested and thus for the most violent impacts, pressure peaks computed by the model are not accurate.
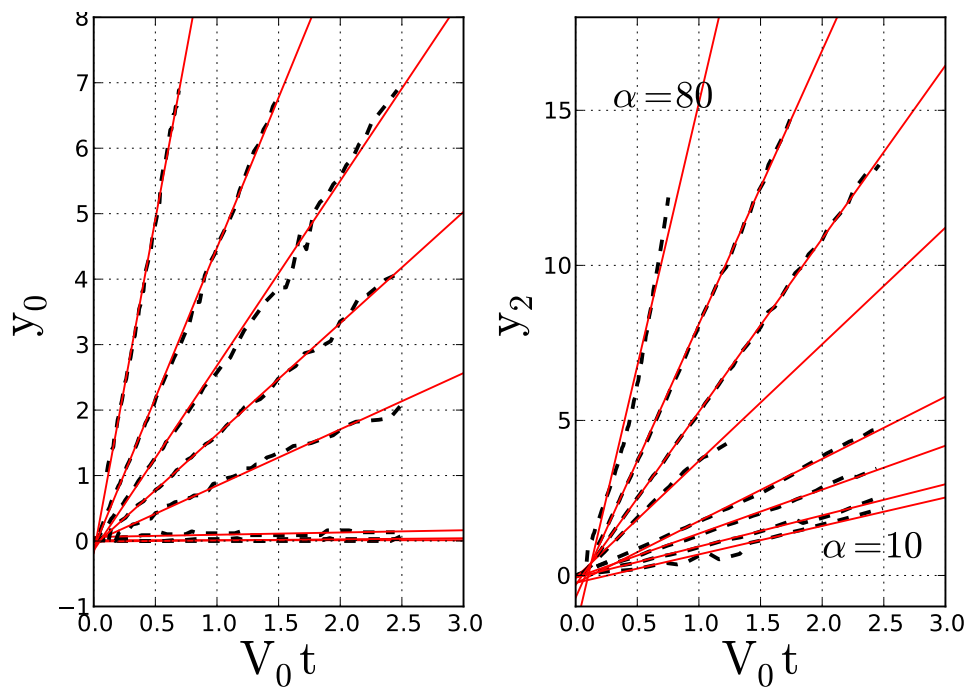

Figure 4: (a) : Evolution of the peak position and wedge through in time - (-) : NS-VOF results, $(-)$ : Linear interpolation, $(--): \mathrm{Wu}(2007)$

Computed positions $y_{0}(t)$ and $y_{2}(t)$ are presented on figure 4 . We recall that they correspond to the position of the peak and the highest free surface point on the wall. A linear regression has been performed using the least square method. Corresponding residuals are assessed to $10^{-4}$ for $\alpha=10^{\circ}$ and $10^{-1}$ for $\alpha=80^{\circ}$, demonstrating that the positions are a linear function of time. As expected, these particular points move with constant velocity respecting the self-similar properties. 


\section{Derivation of analytical laws}

Taking into account that pressure peaks seems not accurate for high values of $\alpha$, whereas positions of pressure peak and $A$ remain valid, we have tried to develop a relation that allows to deduce the peak from these specific positions.
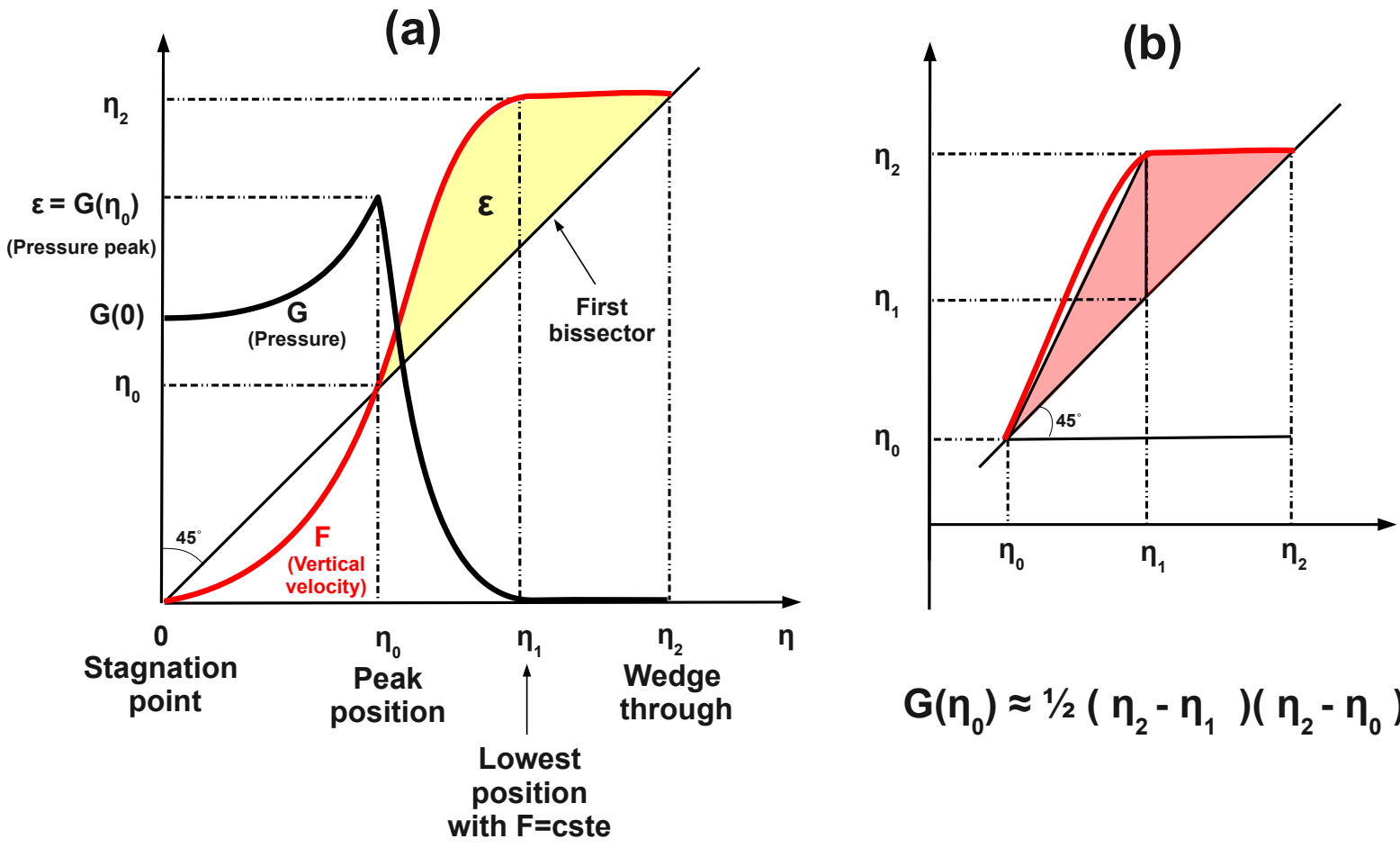

$$
G\left(\eta_{0}\right) \approx 1 / 2\left(\eta_{2}-\eta_{1}\right)\left(\eta_{2}-\eta_{0}\right)
$$

Figure 5: (a) : Schematization of velocity and pressure profiles on the wall - (b) : Pressure peak assessment according to specific positionon the wall

The details of the theory can be found in Mokrani (2012). An intermediary position $\eta_{1}$, corresponding to the lowest position from which the pressure is zero (cf. figure 5a) is defined. By integrating (1), we can show that the peak magnitude equals the area represented in yellow on figure 5a. We then approximated this area by the triangular area represented on red on figure $5 \mathrm{~b}$ which gives :

$$
P_{\max }=\frac{1}{2}\left(\eta_{2}-\eta_{1}\right)\left(\eta_{2}-\eta_{0}\right) P_{0}
$$

Computed positions $\eta_{0}, \eta_{1}$, and $\eta_{2}$ have been interpolated by second order polynomial functions in tan $\alpha$, in order to compare our result with Greco (2001), who provides a similar analytical law for $\eta_{0}$. Results are presented on figure 6. Subsequent expressions and corresponding residuals $(R)$ are :

$$
\begin{array}{cc}
\eta_{0}=-0.007 \tan ^{2} \alpha+1.99 \tan \alpha-0.85 & R_{0}=0.054 \\
\eta_{1}=0.08 \tan ^{2} \alpha+1.57 \tan \alpha+0.21 & R_{1}=0.024 \\
\eta_{2}=0.21 \tan \alpha+2.3 \tan \alpha+0.95 & R_{2}=0.0062
\end{array}
$$

Greco (2001) derived the exact solution of $\eta_{0}$ for small value of $\alpha: \eta_{0}=2 \tan \alpha$. Contrary to Greco (2001), we obtained a constant term in (3), which means that it exists a minimum inclination from which a pressure peak is detected on the wall. Previous results have shown that this minimum value is about $\alpha \sim 40^{\circ}$. The present results suggests that the velocity of the peak position also evolves quasi linearly in $\tan \alpha$ (the coefficient in $\tan ^{2} \alpha$ being low) for high interface inclinations and thus for more violent impacts. On the contrary, $\eta_{2}$ has a significant contribution of $\tan ^{2} \alpha$. 

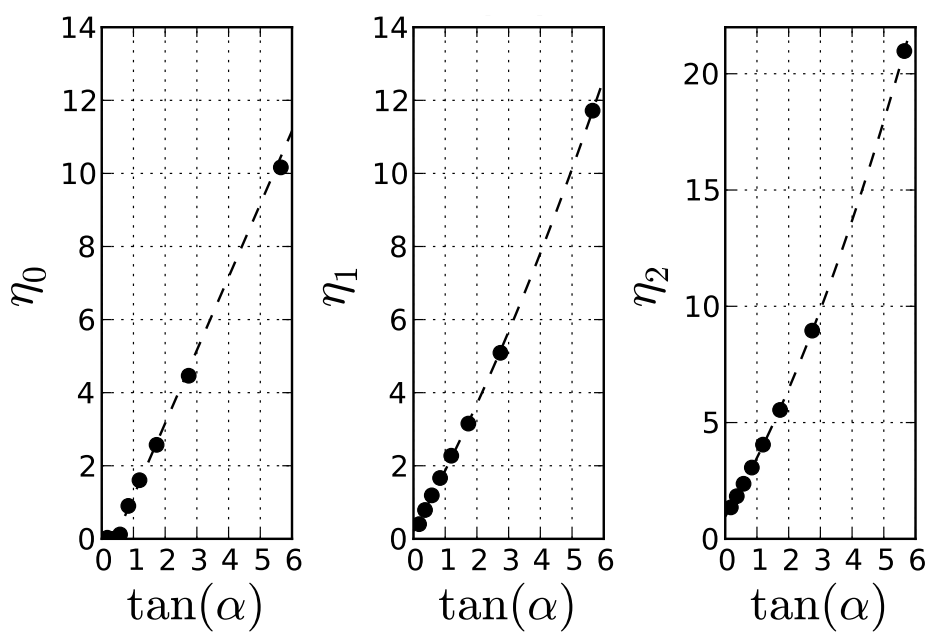

Figure 6: Position $\eta_{0}, \eta_{1}$, and $\eta_{2}$ numerically obtained (•) and corresponding extrapolations functions (dashed lines)

By using expressions (3), (4), and (5) in equation (2), we obtain an semi empirical law for the pressure peak in the wedge impact case:

$$
\frac{P_{\max }}{P_{0}}=0.01 \tan ^{4} \alpha+0.1 \tan ^{3} \alpha+0.38 \tan ^{2} \alpha+0.77 \tan \alpha+0.56
$$

Expression (6) is plotted in green dashed line on figure 7. Zhang et al. (1996) computed pressure peak magnitude for similar case, using a B.E.M. method. Our analytical law agrees well with there results and also with the values obtained in $\mathrm{Wu}$ (2007), for low as well as for high inclinations. For example, for $\alpha=80^{\circ}$, expression (6) estimates the pressure peak within $7 \%$ of accuracy compared to Wu (2007)'s value. Relation (6) shows that the peak magnitude increases strongly with higher angles for high inclinations [as $0.01 \tan ^{4}(\alpha)$ ]. To a certain extend, this characteristics is one reason for the high sensitivity of the pressure peaks observed in numerical simulations (e.g., Abadie and Mokrani (2012)) or in experimentations.

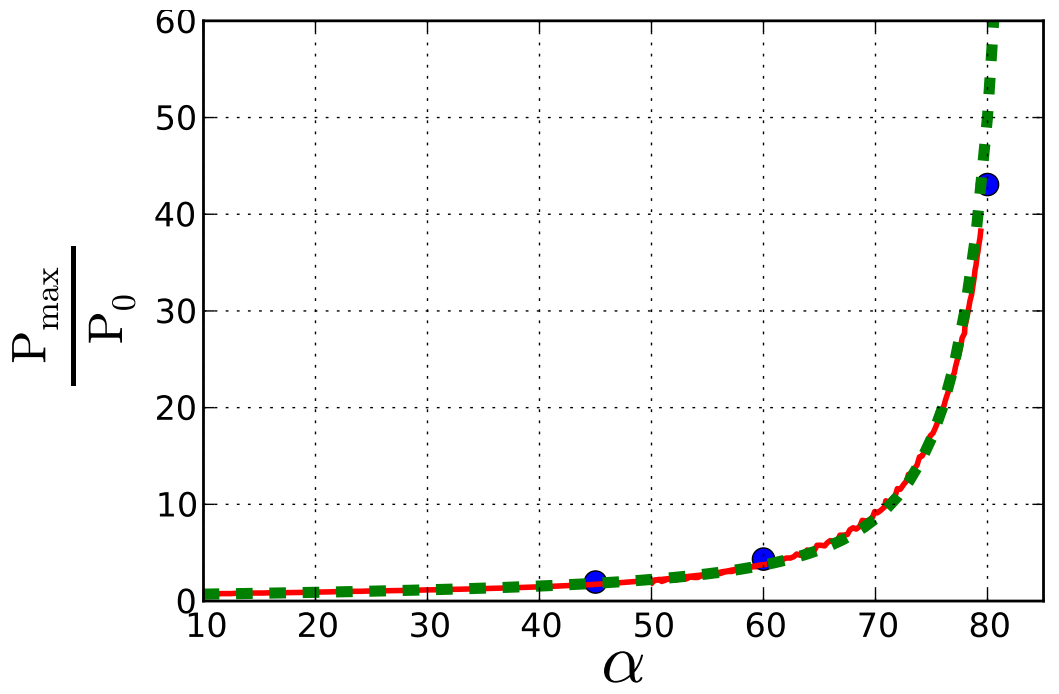

Figure 7: Pressure peak magnitudes according to $\alpha-(\bullet):$ Wu(2007), (-) : Zhang et al. (1996), (- -) : Analytical law (6)

\section{APPLICATION TO A PLUNGING WAVE IMPACT}

In this part, we apply (6) in the case of a plunging wave impact. As previously mentioned, the jet impact of the plunging breaker is interpreted as a succession of elementary wedges impact. The analogy is sketched on figure 8. $\alpha_{C}$ and $\beta_{C}$ denote the instantaneous inclinations respectively associated to the superior and inferior triangular jet. As illustrated in figure 8 , the idea is to estimate pressure peak values by using relation (6). But before that, 
we will use it the other way around to estimate where the equivalent angles have to be placed exactly on the free surface.

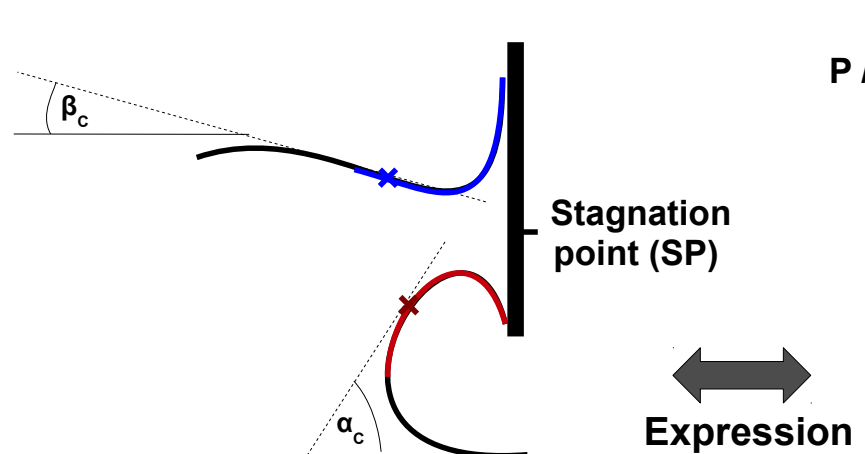

(6)

(a)

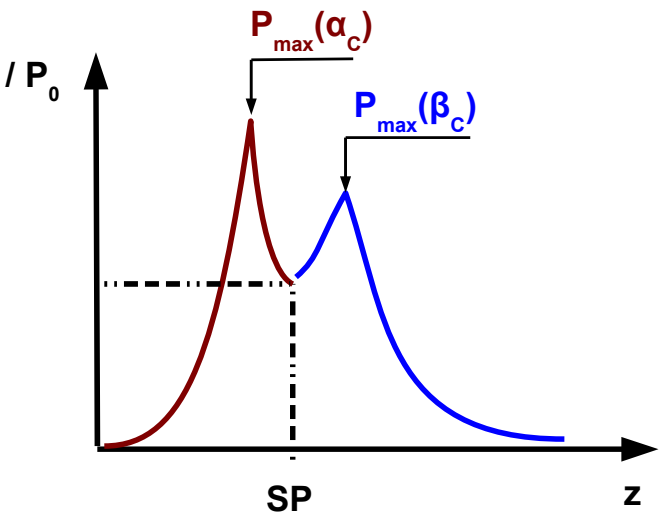

(b)

Figure 8: Method used ot analyze dynamical results in a geometrical way - (a) : Equivalent inclination on the interface profile, (b) : Corresponding pressure profile on the wall and pressure peaks obtained from computation

\section{Calculation of the equivalent inclinations}

To simulate the plunging breaker, we consider the problem of a solitary wave propagating over a sloping beach. This case has already been studied by Grilli et al. (1997). Their solution just before impact are used to initialize the Navier-Stokes model. Figure 9 presents a free surface comparison between Grilli's results and the present work at two different stages of breaking. The dynamics of the breaking is very similar in both case which validates our simulation considering B.E.M. results as a reference (several papers already demonstrated the accuracy of this method to describe wave breaking).
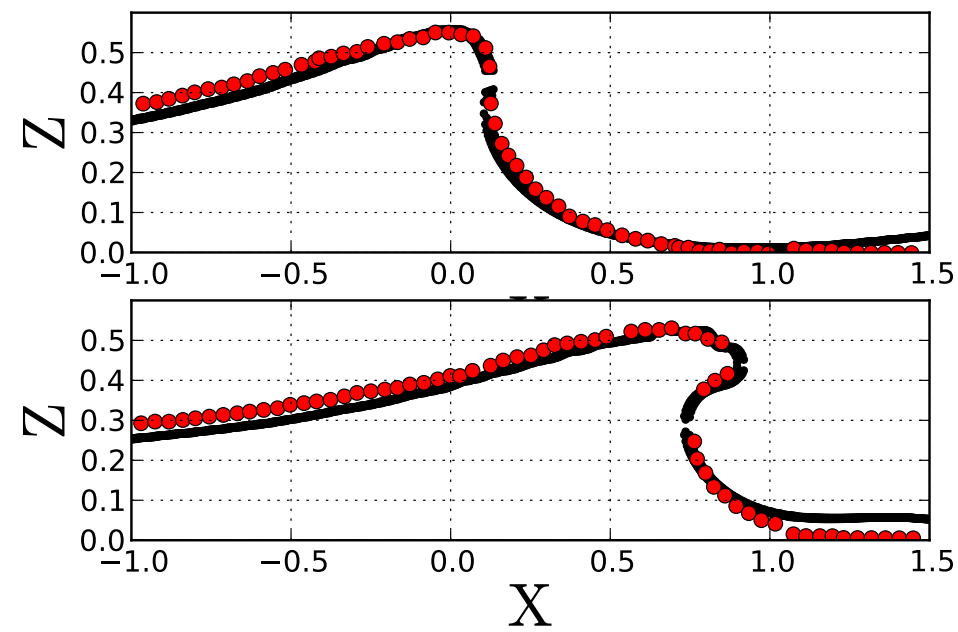

Figure 9: Breaking stage - (- -): Present results, (•) : Grilli et al. (1997)

To simulate the wave impact, the wave breaking simulation is stopped after the jet has developed enough. Then, an obstacle is included in the computational domain and the calculation is restarted. In the case presented, the impact computation has been initialized when the plunging jet velocity was horizontal (similar to Figure 1a). Figure 10 shows that in this case, the stagnation point approximately keeps a constant position on the wall during the impact, similarly to the wedge impact case, justifying the analogy. Note that the wall shape used in the computation and shown in figure 10 allows air leakage and by this, avoid any air entrapment to occur.

At a given time, the pressure peak on the wall is calculated by THETIS. We have verified that the angles remain lower that $70^{\circ}$ so that the results are valid. Then relation (6) is inverted to obtain the angle which would produce the same peak in the wedge impact. Finally, we locate the free surface point that has the corresponding local slope (Figure 11). For this particular case involving a regular interface profile, a unique couple of points exist at each 

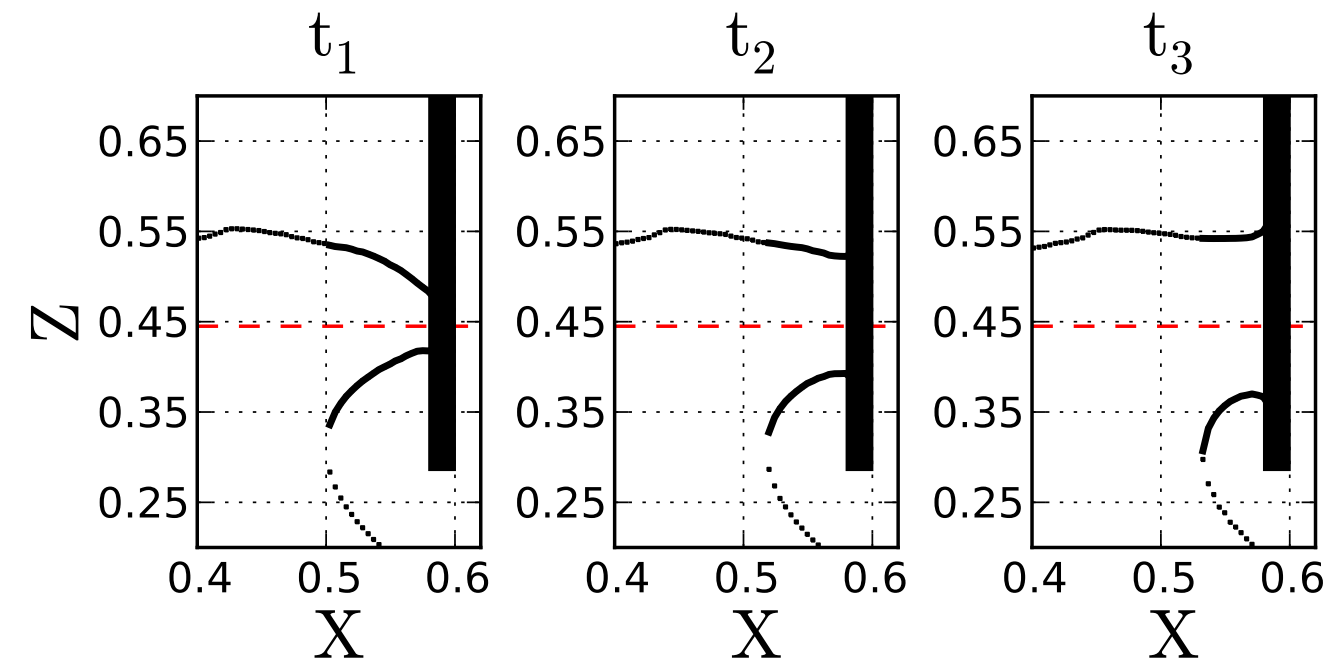

Figure 10: Impact process at three different instants - (- -) : Altitude of the stagnation point

time which is located a bit upstream the free surface local minimum. However the position of both points changes slightly with time as the free surface deforms.

To see whether these points verify any specific geometrical properties, we have compared the position calculated by inverting (6) and the position of the minimum curvature on the free surface (Figure 12). The choice of the minimum curvature seems logical as it may be the location of the free surface the closest to a linear interface. We can see on the figure that both sets of points are very close to each other. This suggests that the local tangent at the minimum curvature point may be used as a proxy to determine the instantaneous impact pressure. This will be verified in the next section.
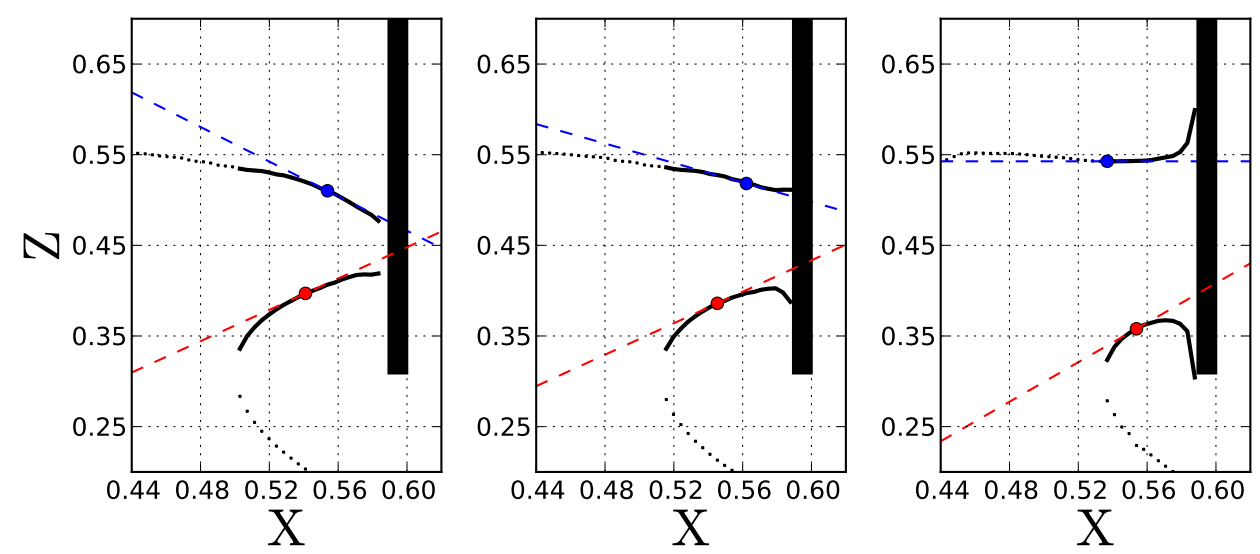

Figure 11: Visualization of point having an equivalent inclinations assessed by (6) - (- -) : $\alpha_{C}$, and (- -) : $\beta_{C}$

\section{First application of the method}

The method is now applied on a new simulation for which a near vertical wave front impacts the vertical wall. This flip-through like impact has been obtained by including an obstacle right before the start of the breaking stage to stop the entire wave motion before the jet develops.

Figure 13a shows snapshots of the interface computed by the modeland the evolution of the minimum curvature point. Based on the previous results, this point is the location where the equivalent angle required in equation (6) 


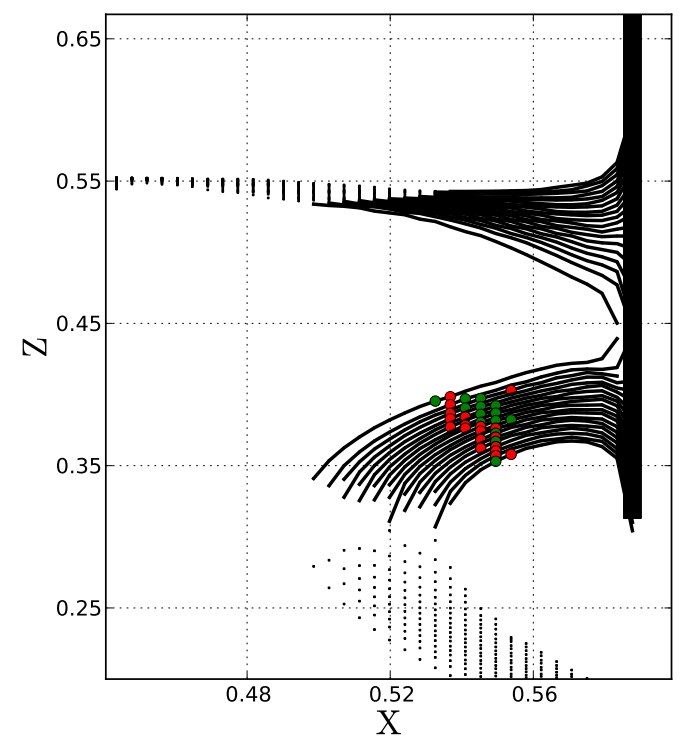

Figure 12: Interface deformation during the impact $(\bullet)$ : Positions of the minimal curving point $(\bullet)$ : Positions of equivalent inclinations

has to be calculated. In figure 13b, we have plotted the time evolution of the pressure peak computed directly by THETIS, together with the approximation of the peak pressure obtained by using equation (6) and the inclination at the minimum curvature point. As observed, the evolution of the pressure peak can be predicted very well during the first instants of the impact. After $\sim 10 \mathrm{~ms}$, this prediction is no longer accurate. This could be due to the error generated by the VOF method (for high inclinations, the curvature assessment becomes more difficult). The last point could however be improved by using finer mesh grids. This will be investigated in further works.

(a)

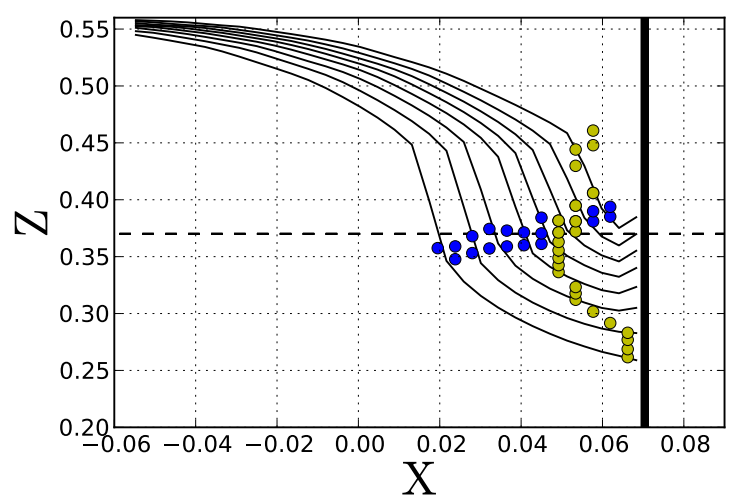

(b)

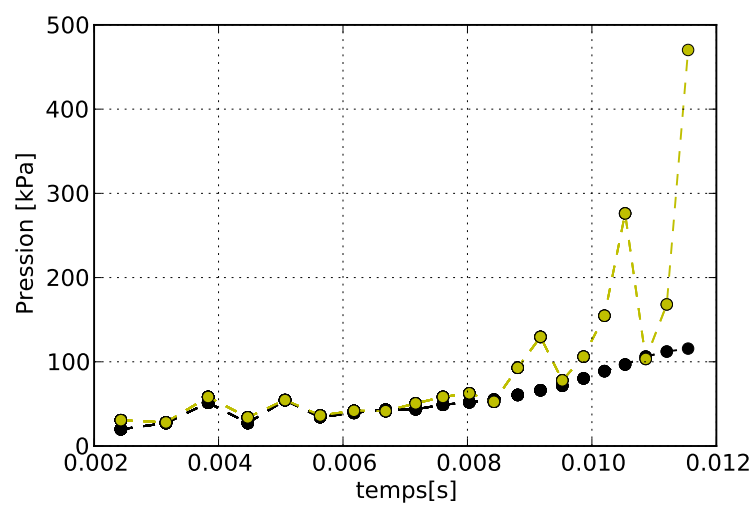

Figure 13: Interface profiles during a flip through process (a) : Evolution of the minimum curvature point ( $)$ and the point having maximal inclination $(\bullet)$ - (b) : Comparison between the time evolution of the pressure peak numerically assessed $(\bullet)$ and predicted by using the inclination at the minimum curvating point and expression (6) ( )

\section{CONCLUSION}

This study focuses on the wave shape effects on impact dynamics with the help of a Navier-Stokes model (THETIS). We have developed a method to calculate the instantaneous pressure peak generated by a breaking wave knowing the free surface evolution. The main idea is to assimilate the breaking jet to two symetrical wedges. For the wedge case, we have developed a semi empirical law relating pressure peak and incident angles based on numerical simulations. We have also shown that our numerical simulations are accurate only for incident angles lower than $80^{\circ}$. In the first simulation involving a breaking wave, we have determined, by that inverting our law 
that the position where the equivalent angle has to be taken corresponds to the minimum curvature point on the free surface. For another simulation, we have then tried to assess the pressure peak by using our relation which only uses the free surface information. The comparison with the direct computation using the Navier-Stokes model shows that the method can work but needs further investigations to be more deeply validated.

\section{REFERENCES}

S. Abadie and C. Mokrani. On the influence of breaking wave local geometry on impulsive loads. Proceeding of the International Offshore and Polar Engineering Conference, 2012.

S. Abadie, J.P. Caltagirone, and P. Watremez. Splash-up generation in a plunging breaker. Computational fluid mechanics, Série II.b:553-559, 1998.

M.J. Cooker and D.H. Peregrine. Pressure-impulse theory for liquid impact problems. J. Fluid Mech., 297: 193-214, 1995.

E. Cumberbatch. The impact of a water wedge of a wall. J. Fluid Mech, 7:353-374, 1960.

Z.N. Dobrovol'skaya. On some problems of similarity flow of fluid with a free surface. J. Fluid Mech, 36:805-529, 1969.

M. Greco. A Two Dimensional Study of Green-Water Loading. PhD thesis, Faculty of Marine Technology, Trondheim, 2001.

S.T. Grilli, I.A. Svendsen, and R. Subramanya. Breaking criterion and characteristics for solitary waves on slopes. Journal Of Waterway, Port, Coastal and Ocean Engineering, 123(3):102-112, 1997.

P. Hull and G. Muller. An investigation of breaker heights, shapes and pressures. Ocean Engineering, 29:59-79, 2002.

P. Lubin, S. Vincent, S. Abadie, and J.P. Caltagirone. Three dimensional large eddy simulation of air entrainment under plunging breaking waves. Coastal engineering, 53:631-655, 2006.

C. Lugni, M. Brocchini, and O.M. Faltinsen. Wave impact loads : The role of the flip-through. Physics of Fluids, 18:122101-122118, 2006.

C. Mokrani. Impact de vagues déferlantes sur un obstacle vertical. Modèle théorique et calcul numérique des pics de pression. PhD thesis, Université de Pau et des Pays de l'Adour, 2012.

S. Nagai. Wave forces on structures, academic press, new york. Advances in Hydroscience, 9:253-324, 1973.

G. Pianet, S. Vincent, J. Leboi, J.P. Caltagirone, and M. Anderhuber. Simulating compressible gas bubbles with a smooth volume tracking. International Journal of Multiphase Flow, 36:273-283, 2010.

A.F. Whillock. Measurements of forces resulting from normal and oblique wave approaches to small scale sea walls. Coastal Engineering, 11:297-308, 1987.

G.X. Wu. Fluid impact on a solid boundary. Journal of Fluids ans Structures, 23:755-765, 2007.

D.L. Yougs, K.W. Morton, and M.J. Baines. Time-dependent multimaterial flow with large fluid distorsion. $N u$ merical methods for fluids dynamics, Academic Press, New York, 1982.

S. Zhang, D.K.P. Yue, and K. Tanizawa. Simulation of plunging wave impact on a vertical wall. J. Fluid Mech, 327:221-254, 1996. 